\title{
人工智能化背景下高校人才培养的创新研究
}

边艳华

许昌电气职业学院

DOI:10.32629/er.v2i5.1820

[摘 要] 人工智能技术促进了传统教育的发展, 但在人工智能应用过程中也出现了较多的问题, 如研发投入较少,教育数据的 规范性较差,教师综合素质有待提高等。对此, 政府部门应加大教育领域人工智能的投入,制定细致完善的标准,不断提高教师的 综合素质。

[关键词] 人工智能; 教育领域; 教师综合素质

人工智能在教育领域中的应用已经成为教育行业发展 的主要趋势, 教育人工智能主要实现了人工智能与科学学习 的有机结合, 从而形成集合多个学科的新技术和新系统, 高 校教育教学是人工智能应用的重要领域, 其在人才培养和创 新方面发挥了十分关键的作用。

\section{1 人工智能教育在高校教育应用}

1.1 情感计算可帮助教师掌控课堂

情感计算通常指依靠人力编程, 使机器识别和处理人类 情感的技术。站在知识教学的有效性角度, 人工智能情感计 算几乎能够提高教学的效率, 高校教育教学通常采用大班授 课方式。教师不能全面地了解学生的情感态度, 而人工智能 情感计算能够充分改变这一现状。情感计算技术能够帮助教 师充分了解课堂情况, 及时地了解学生的情感状态, 进而为 教师提供调节学生情绪的有效方法。

1.2 机械学习提高了教育大数据处理的质量和效率

人工智能中, 机器学习是最为核心也是最为重要的技术, 其充分融合了计算机科学和统计学。该技术使计算机可以经 验数据为依据, 改进和完善算法, 进而不断提升性能水平。机 械学习也可以大数据为基础总结规律, 科学预测。在教育领 域应用上述技术, 教师可利用人工智能技术当中的机械学习 技术建模预测学生的行为。机械学习是人工智能技术当中十 分受人关注的技术, 其也广泛地应用在理论内容的学习当中。

应用人工智能技术可分析处理大学生网络信息数据, 人 工智能技术当中的机械学习技术可对不同情景和精度进行 全面分析, 并利用经验数据处理中保证分析的科学性与准确 性。该技术可大量收集教育数据, 同时分析预测学生的状态。

\section{2 基于人工智能的职业素养需求}

2.1 学习能力较强的智能型人才

信息时代知识的获取更加便利, 随着人工智能的迅速发 展, 应用人工智能技术深化了多个行业从业者的学习观念, 增强其迅速聚合知识的能力。现如今, 大数据技术不断发展, 数据容量越来越大, 增长速度越来越快, 同时结构也存在着 十分明显的差异, 其对知识的加工和深度学习均产生了显著 的影响。人才培养的过程中应不断增强多种信息环境背景下 的知识聚合能力。知识聚合主要指以明确事物本质为目标,
利用系统思维将多个零散的知识形成完整的知识结构或知 识框架, 或者也可合理利用知识单元之间的联系, 以元数据 层面和社会标签、关联数据及现有的关系等对知识组织方法 的框架予以深入研究。这也充分顺应了人工智能联结主义的 基本要求, 其也是人工智能不断发展的今天, 人才培养中的 核心智能素养。

\section{2 对多领域融会贯通的复合型人才}

人工智能不断发展的今天, 科技素养与人文素养之间的 有机结合, 文理知识的相互渗透是发展的重要趋势和方向。 人工智能涉及到多门学科, 而且其涉及的领域特相对较广, 具有较强的交叉性。其对从业人员的跨界融合能力及灵活应 用多领域知识和技能的能力提出了较高的要求。以解决问题 为主要的目标, 运算和执行是智能机器的主要优势, 而思考 决策是人独到的优势。在人工智能产业发展中, 从业者需更 加深刻地理解跨行业、多领域的融合, 一方面要不断增强直 觉灵感思维, 另一方面还应增强科技实证的能力, 从而真正 地实现夸张专业合作, 推动创新, 增强人才解决问题的能力。 人工智能日益发展的当下, 在产业融合发展中也要不断提升 其转化和反思能力。

2.3 德才双全的社会型人才

人工智能技术就是模仿人类的一种智能技术, 其以程序 设计为基础, 多种形式的智能机器均能够实现人脑的部分功 能, 并且结合实际进行功能延伸。以功能、智能、智力和智 慧的智能标准来划分, 人工智能处于第二梯级, 也就是利用 人工创建的算法不断提高任务执行的准确性和效率。但是, 人工智能无法应用于复杂判断、高级创造和深刻洞察等智力 加工领域。如从现代智慧心理学的角度来看, 人工智能依然 是一种小智慧, 只能实现部分认知智慧, 而无法体现其理论 与实践智慧。另外, 在应用的过程中也不能很好地解决与自 我高度密切相关的不确定事件及问题。出现这一现象主要是 由于人工智能没有情感, 也没有自我意识。智慧是聪明才智 和完备的道德的融合, 与人工智能相比, 人在情绪情感、公共 利益、社会合作和社会责任方面均有着较大的智慧, 这也是 人工智能时代所需要的社会型职业素养, 是人工智能技术无 法跨越伦理的主要因素。 


\section{3 人工智能背景下的高校人才培养策略}

3.1 由静态教育模式转向交互型教育模式

在人工智能不断发展的时代, 人们可十分便捷地获取理 论知识。传统的灌输填鸭式的教育模式已经无法满足教育发 展的需要。因此其也逐渐被以学习者为核心的新型教育理念 和模式取代。高校人才培养观念也发生了较大的变化, 从简 单的为社会提供劳动力转变为社会提供智能交互的学习体 验。在体验式的教育服务发展中, 高校应当更加关注并重视 学生综合性发展的综合价值, 激发学生自主学习的意识, 从 而培养学生解决问题的思维, 让其学会创新, 形成合作共赢 的精神。此外, 利用程序性的知识获得知识练习和实践的平 台, 可以不断增强学生的智能思维和创造能力。高校的学习 者需要积极转变学习的方式, 从以往的死记硬背的静态学习 模式转变为高度重视知识聚合的交互型学习模式, 加大学习 的深度。

高校学生在人工智能不断发展且学科专业跨界融合不 断加深的背景下, 要增强自身探索不同学科知识和新旧知识 之间联系的能力, 从而揭示事物的本质, 建立更加积极和有 意义的知识框架。学生在学习中也可利用人工智能界面的交 互作用和学习资源、学习伙伴及教师的信息交互, 真正地实 现知识结构中新旧知识和概念的交互、转变及发展。再者, 高校需不断发展人工智能+的教育环境, 建设以大数据为基 础的云端学习平台, 进而促进智能素养的融会贯通。且充分 尊重学生在教育教学中的地位, 不断推广教学方法和教学评 价的变革, 从而提供更加准确、多元和个性化的教育服务。 不仅如此, 还应在校园硬件建设的过程中配套推动人工智能 技术的开发与应用, 建设更加安全便捷的智能校园, 不断创 建交互性的学习环境。

3.2 由培养一专多能型人才转变为培养跨界复合型人才 在高校的建设和发展中, 其要为区域产业经济和社会建 设发展输出大量的智能人才, 高校专业建设调整和产业结构 的升级也需保持协调的关系。当前大物移云信息技术不断发 展, 新型产业和智能改造后的制造业服务业也得到了前所未 有的进步。高校在人才培养中需积极转变传统的理念和思维, 不断发展人工智能 + 的复合型人才培养体系。

在人工智能基础上, 产业边界也在不断扩大, 与传统产 业的融合深度也在不断增加。因此, 高校需建立跨界开放的
人才培养体系, 全面极推动学科和专业的融合, 在教育教学 的过程中高度重视跨界和联合培养, 从而建立完善的学科学 习保障机制和专业课程体系, 将上述体系与人工智能课程体 系有机结合, 建立起更加完善的人才培养和保障制度。在跨 界复合型的智能人才培养中, 每个学生均可成为社会发展所 需要的智能型人才。

3.3 从培养工具型人才转变为培养智慧型人才

工具性的人才培养观念无法顺应人才全面发展的基本 要求。互联网、计算机和人工智能均是一种十分常见的现代 工具, 在高校人才培养的过程中, 不可将人才视为职业工具, 而是要不断增强学生创造和使用工具的能力。所以, 人工智 能只是高校培养人才的一种方式和技术, 学生顺应智能时代 发展, 展开高效的学习, 不断提高学生的系统思维和创新能 力, 成为了高校人才培养的主要目标。与人工智能相比, 高校 需不断培养智慧型人才。职业能力只是学生综合发展中的一 个方面, 其同样也展现在自我认知、组织管理、决策能力、 批判思维、情绪管理和技术创新等多个方面。虽然人工智能 技能培训与应用的普及, 可增强学生的认知能力, 但是人工 智能在情感、伦理等多个方面均存在着明显的不足, 这也需 要高校在日后的人才培养中高度重视德育教育, 始终坚持德 才兼备和全面发展的智慧型人才培养导向, 防止在人才培养 中单纯地关注就业, 同时还要将人文教育、社会责任感的培 养融入到智能人才培养的过程中, 进而培养职业能力和道德 素养均在较高水平之上的全面发展型人才。

\section{4 结束语}

人工智能成为了当前高校教育中主要的发展方向, 其也 对高校人才培养提出了更高的要求。高校在人才培养中, 需 断转变以往的人才培养理念和培养模式, 培养出更多顺应人 工智能时代的智能型和智慧型人才, 以期实现人才的多方向 发展。

\section{[参考文献]}

[1]连悦寒.人工智能对传统会计的影响 [J].中国市 场,2019(16):192-193.

[2]马楠,刘元盛, 李德毅.智能时代与大学创新人才培养 [J].高等工程教育研究,2017(06):164-167.

[3]周晓峰. 发展人工智能更要超前布局 [N]. 青岛日 报,2019-06-05(006). 